Atom Probe Tomography Defines Mainstream Microscopy at the Atomic Scale

Thomas F. Kelly ${ }^{1}$, Keith Thompson ${ }^{1}$,

Emmanuelle A. Marquis ${ }^{2}$, and David J. Larson ${ }^{1}$

${ }^{1}$ Imago Scientific Instruments Corp., Madison, WI

${ }^{2}$ Sandia National Laboratory*, Livermore, CA

\title{
Introduction
}

When making a sculpture, it is the eyes that guide the hands and tools and perceive the outcome. In simple words, "in order to make, you must be able to see." So too, when making a nanoelectronic device, it is the microscope (eyes) that guides the process equipment (hands and tools) and perceives the outcome. As we emerge into the century of nanotechnology, it is imperative that the eyes on the nanoworld provide an adequate ability to "see." We have microscopies that resolve $0.02 \mathrm{~nm}$ on a surface (scanning tunneling microscope (STM)) or single atoms in a specimen (atom probe tomographs (APT) and transmission electron microscopes (TEM)). Surely, that must be good enough. Well, we need even better. The ideal microscope for nanotechnology would reveal the position and identity of all atoms in a specimen for as far as the user wished to see in any material with high sensitivity. Is this realistic? It is not possible in any scanning probe instrument or electron microscope. However, we come close to this ideal in today's atom probes.

Atom probe tomography can provide the position and identity of atoms (more specifically, isotopes) in a 3-D volume that is greater than 100 by 100 by $500 \mathrm{~nm}$ with a resolution on the order of $0.2 \mathrm{~nm}$. Half of all the atoms are detected with equal efficiency for all species and with analytical sensitivity in the 10-part-per-million range. With

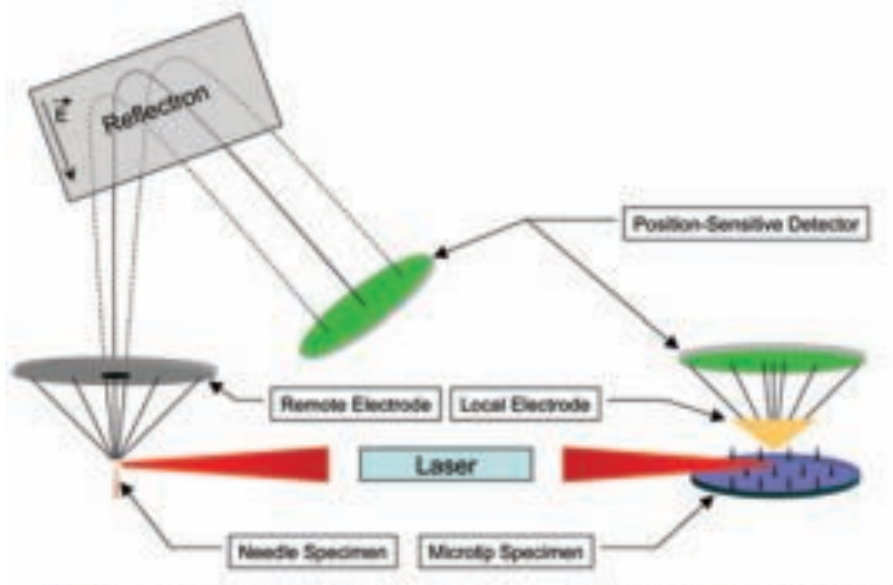

\section{Rellectren-compensated 3OAP}

Figure 1. Comparison of a LEAP to standard (remote) electrode atom probes. The LEAP $3000 X$ incorporates a sophisticated laser-pulsing system focused automatically to very small and exacting dimensions.

the introduction of commercially-available laser-pulsed atom probes, most solid materials, even organics, can be analyzed.

At this time, the atom probe does not provide direct imaging of defects (TEM), does not provide a low magnification survey of a specimen (SEM, TEM), does not directly measure physical or chemical properties (TEM, SPM), is not sensitive to surface reconstructions or reactions (SPM, AFM), and is not yet ready for complex organics and biologicals (SEM, TEM.) For most aspects of atomic-scale analytical microscopy however, atom probe tomography will be the simplest and most powerful route to high quality results. Today's commercial instru-

* Sandia is a multiprogram laboratory operated by Sandia Corporation, a Lockheed-Martin Company, for the United States Department of Energy National Nuclear Security Administration under contract DE-AC04-94AL85000.

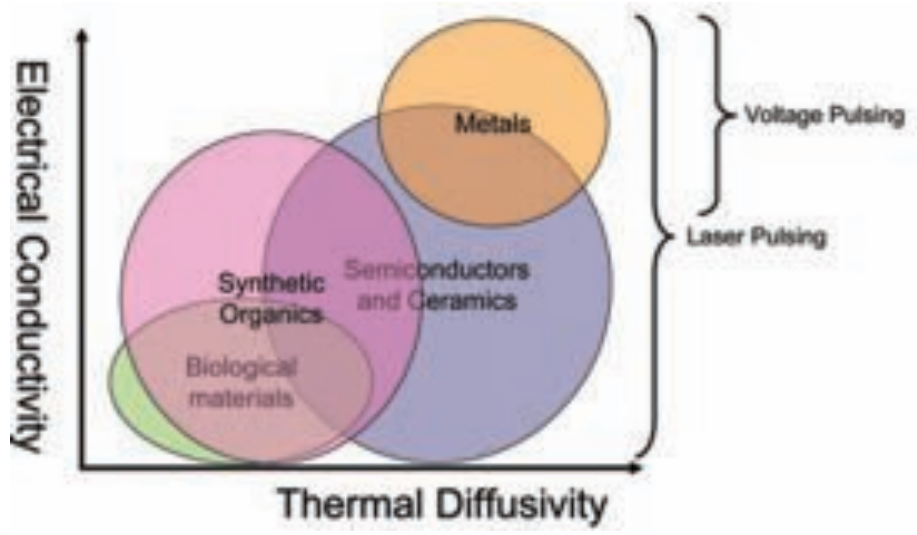

Figure 2. The effect of laser pulsing on the addressable materials space. Only metals and a few semiconductors could be analyzed with voltagepulsing. Laser pulsing opens the space up to all materials.

ment is easy to use, highly automated, fast, and reliable. Facilities that specialize in nanoscale characterization must consider the essential complementarity of the atom probe with the other high resolution microscopies. It is in many ways defining the state of the art in mainstream microscopy today.

\section{How an atom probe works}

An atom probe is an integrated point-projection microscope and time-of-flight mass spectrometer. The atom probe achieves this by recording positional information, namely, two-dimensional hit position on the detector and one-dimensional sequence number, along with time of flight for each ion analyzed. Ions field evaporated from the needle-shaped-specimen surface during atom probe analysis create a highly-magnified projection of the atomic-scale structure of the specimen surface on the detector, Figure 1. Further, since atomic layers erode predictably from the specimen surface, evaporation sequence number can be used to calculate an ion's position in $\mathrm{z}$ (direction normal to the specimen surface) in the original specimen with high precision.

The technique offers quantitative 3-D compositional imaging and analysis. Further, atom probes detect all elements, isotopes, and higher-mass molecular ions. The unique power of atom probe microscopy lies in its ability to tie compositional information to structure. Other techniques can determine physical structure at sub-nm levels, but only atom probe has the capacity for compositional identification (and quantification) at sub-nm levels as well.

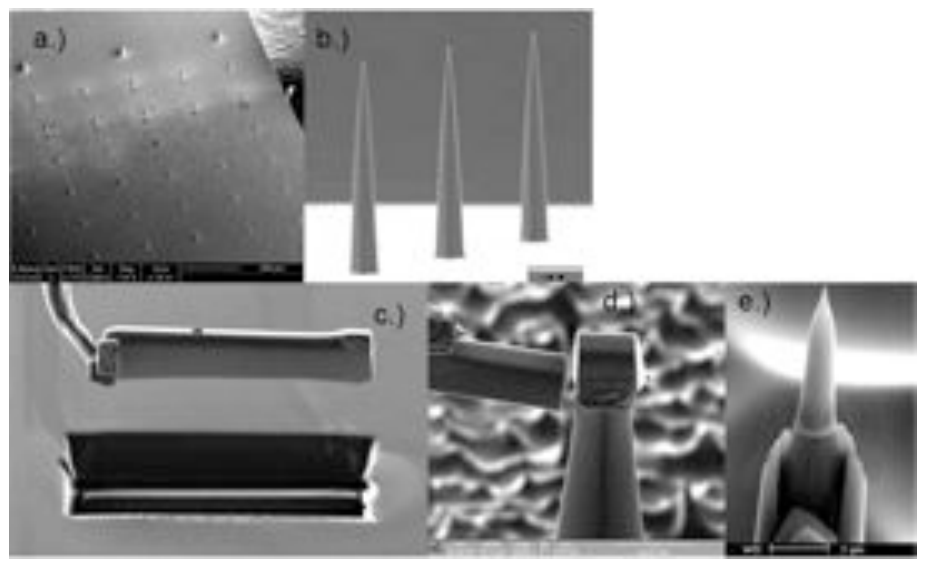

Figure 3. a) low magnification view of a microtip array in silicon, $b$ ) higher magnification view of individual microtips, c) a coupon being lifted out of a silicon wafer, d) a piece of the coupon attached to a microtip, $e$ ) the sharpened piece ready for LEAP analysis. Coupons have been extracted and mounted to make 12 specimens with an average time of 20 minutes per specimen. 


\section{Quantitative 3D Compositional Imaging and Analysis with Sub-nm Resolution}

\section{Semiconductors}

\section{Data Storage}

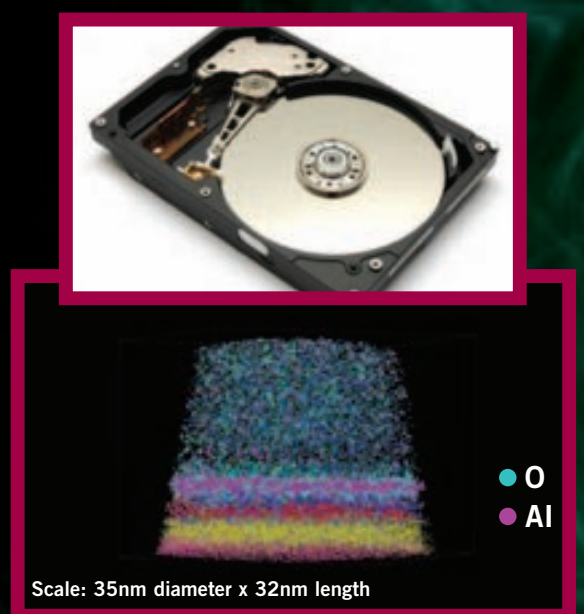

3D Compositional Analysis of Nanomagnetic Materials

- GMR/TMR Stacks

- Buried Oxide Layers

- Interface Layer Roughness

- $\mathrm{CCP} / \mathrm{CPP}$ Structures

Imago will offer an atom probe workshop Wednesday, 5-7 at M\&M. Admission is free but reservations are required. Contact information@imago.com.

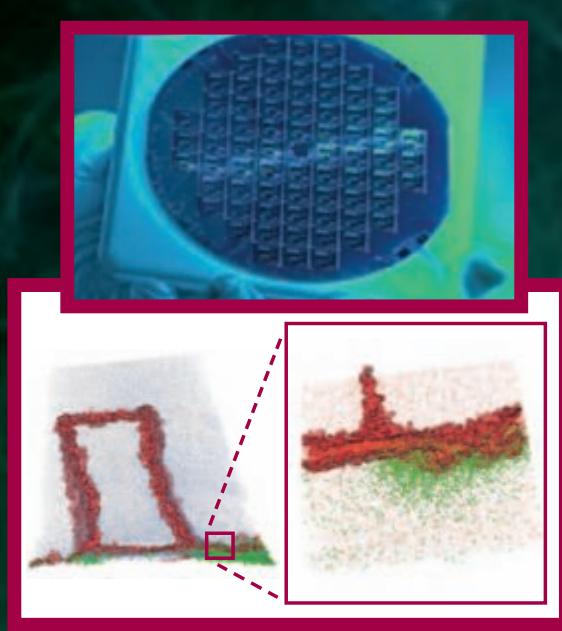

3D Dopant and Source/Drain Interface Analysis

- USJ Dopant Profiles

- Lateral Diffusion (Straggle)

- Silicon Germanium

- High-k Dielectrics

- FinFET Structures

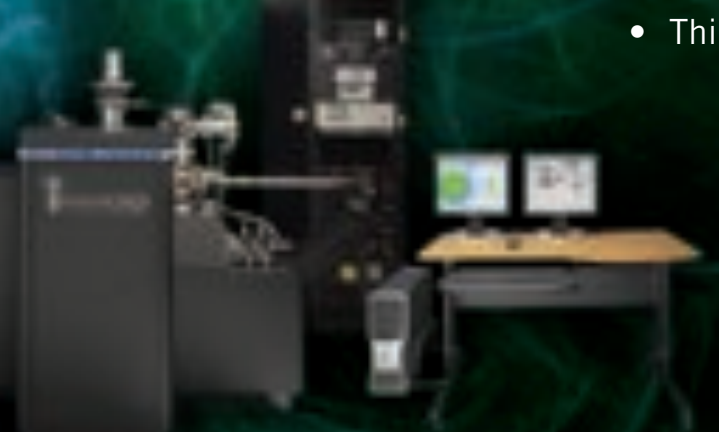

\section{Advanced Materials}

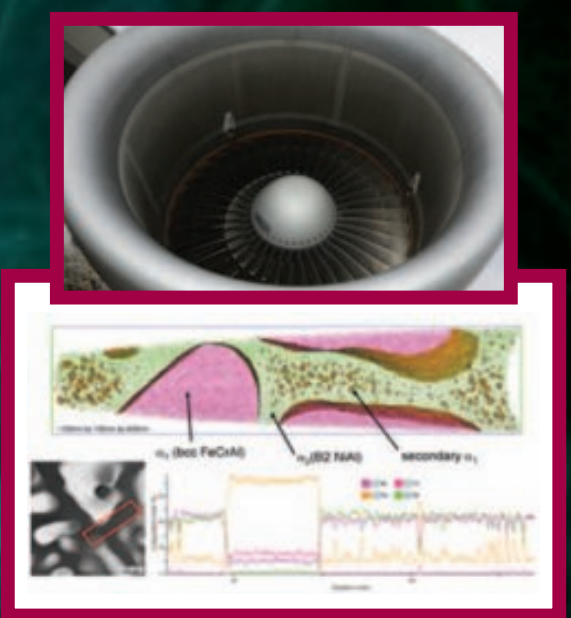

3D Mapping of Micro- and Nano-Scale Segregation

- Phase/Grain Boundary Analysis

- Precipitates

- Nuclear Materials

- Thin Metal Films

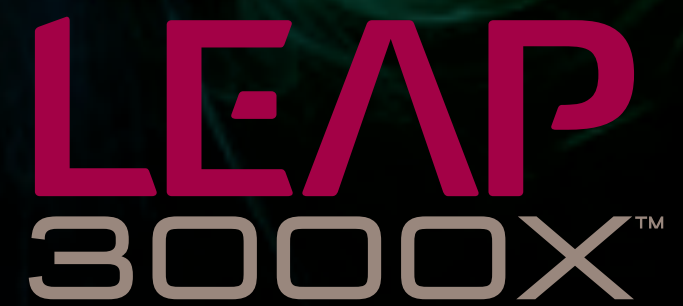

EXTREME METROLOGY AT THE NANO-SCALETM

For more information, visit us online at www.imago.com. 

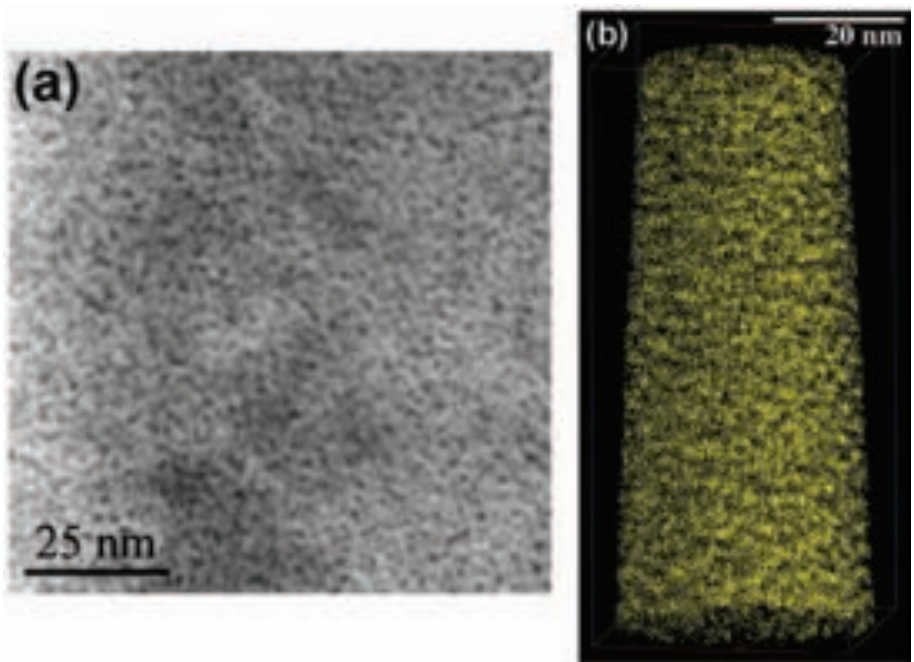

Two approaches to atom probe construction are illustrated in Figure 1, the reflectron-compensated 3-dimensional atom probe (3DAP) and the local electrode atom probe $\left(\right.$ LEAP $\left.^{\circledR}\right)$. In order to measure a time of flight, the field evaporation process must be pulsed so that the departure time can be known. Since field evaporation rates increase with applied field and with temperature, it is possible to pulse either the applied electric field (applied voltage on specimen) or the specimen temperature. In both cases, this must be done in a fraction of a nanosecond. In field pulsing, the departing ions acquire a range of energy due to the pulsed field and this results in poor mass resolution unless something is done. Reflectron-compensated 3DAPs use an electrostatic mirror (reflectron) to time focus the ions on the detector. LEAPs shield the ions from the time-varying fields with the local electrode and achieve good mass resolution by preventing the energy spread from developing. In laser pulsing, the electric field is static and the energy spread from the thermal pulse is negligible so high mass resolution can be readily achieved.

The local electrode is a hollow cone-shaped metal piece that has a small aperture (hole) at its apex. During atom probe operation, the local electrode is positioned close or "local" to the specimen, with the electrode's aperture centered over the specimen tip, (Figure 1). Imago has developed a voltage-pulsed local electrode atom probe, the LEAP $3000^{\text {Ts }}$, and a laser-pulsed and voltage-pulsed version, the LEAP $3000 \mathrm{X}^{\mathrm{sm}}$. Both forms of pulsing are available on a LEAP $3000 \mathrm{X}$ and are selectable in software.

The original LEAP system used an innovative form of voltage pulsing that achieved two orders of magnitude greater pulse repetition frequency $(200 \mathrm{kHz})$ than previous atom probes (1 to $2 \mathrm{kHz}$ ). However, good electrical conductivity in the specimen is required for voltage pulsing, Figure 2. Laser pulsing makes it possible to pulse materials with any degree of electrical conductivity. The challenge in developing a laser-pulsed atom probe was to create thermal pulses of sub-nanosecond duration at greater than $100 \mathrm{kHz}$ repetition rate that would work with poor thermal conductors, Figure 2 . By focusing the laser energy onto a small volume of the specimen ( $<5$ micron length), materials with low thermal diffusivity may be analyzed.

Imago's LEAP 3000X is the only laser-pulsed atom probe that can analyze microtip arrays.[3] The importance of this ability to analyze microtips lies in the way specimens may be configured. As shown in Figure 3, arrays of microtips may be fabricated either from the material to be studied or from substrate materials by, for example, MEMS processing methods. Such microtip arrays can serve many purposes: 1) they may be analyzed directly after some form of processing (thermal, implantation, etc.), 2) they may be coated with thin films of inorganics or organics, 3) they may serve as holders for coupons extracted from wafers or other bulk materials (including biological materials). Coupon extraction methods make it possible to prepare specimens from a very small volume of material $(<1 \mu \mathrm{m} 3)$ from a wide variety of materials. As shown in Figure 3, small extractions may be mounted on a microtip and an entire array of microtips may be populated. The semiconductor and magnetic applications below were both prepared as microtips and focused ion beam milling was used to sharpen the samples [4].

\section{Applications}

\section{Al-Ag Alloy}

Atom-probe tomography has revolutionized the study of metallic alloys by allowing the direct three-dimensional observation of atomicscale phenomena such as solute interaction and clustering that occur during the early stages of alloy decomposition. For instance, in the phase-separating Al-Ag system, intermediate metastable precipitates (Guinier-Preston (GP) zones) are first formed. The exact structure of the GP zones (ordering, Ag concentration) and the existence of temperature-dependent phases (ordered at low $\mathrm{T}$ and disordered at high $\mathrm{T}$ ) is still a matter of debate and the numerous experimental studies published on this system have yet to concur [5-9]. Atom probe tomography can provide clues on the decomposition process through the analysis of the temporal evolution of the Ag atoms' spatial distribution.

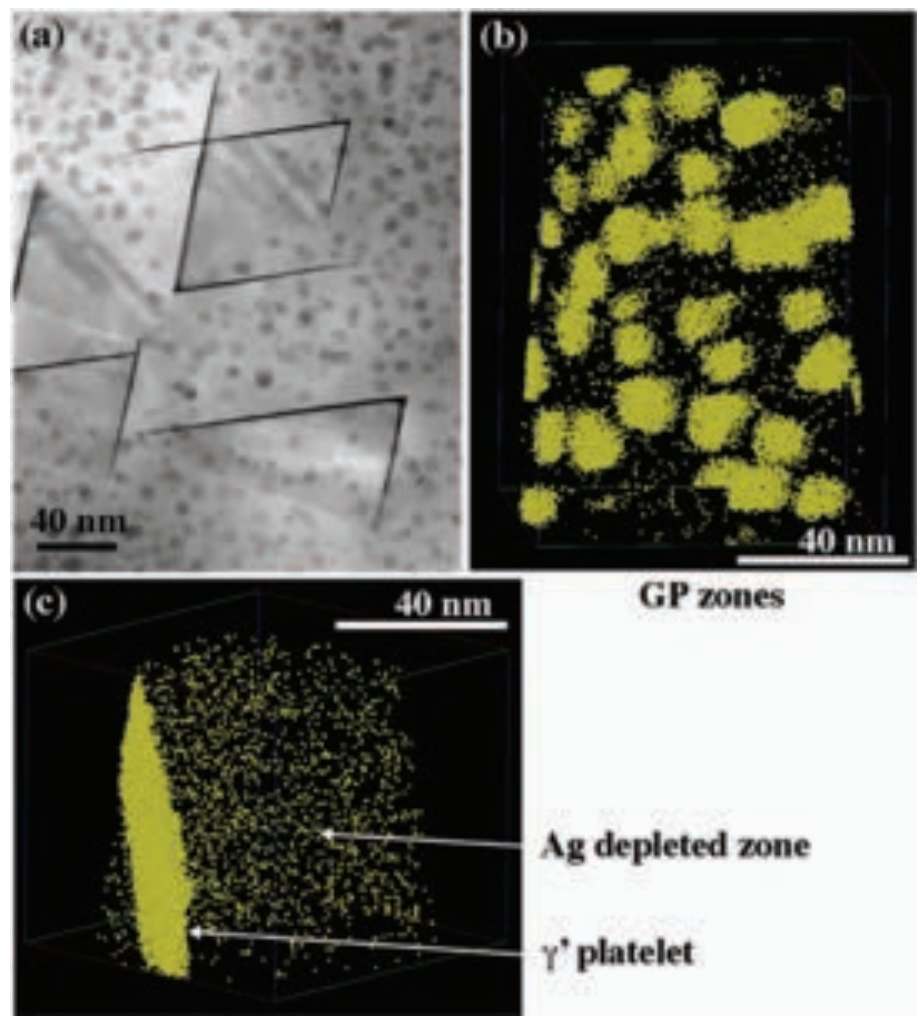

Figure 5. Transmission electron microscopy and local electrode atom analysis data obtained from an Al-3at.\%Ag alloy after aging at $130^{\circ} \mathrm{C}$ for 450h. The microstructure is comprised of faceted GP zones and $\mathrm{g}^{2}$ platelets. A Ag-depleted zone is visible in both the TEM (upper left) and LEAP data (lower) images. Ag atoms shown in yellow.

Specifically, an Al-2.7 at.\% Ag alloy was quenched from $560^{\circ} \mathrm{C}$ in ice water, subsequently aged at $130^{\circ} \mathrm{C}$. For illustration purposes, Figures 4 and 5 are two snapshots of the structure at different aging times. The as-quenched alloy already exhibits phase separation with the appearance of Ag-rich clusters (Figure 4). The clusters do not have a precise shape at early times but evolve to spherical precipitates after 20 hours and eventually become faceted as shown in Figure 5a after aging for 450 hrs. At this longer aging time, $\gamma$ ' platelets (that will eventually evolve into 


\section{e-RAM}

\section{Electron Roughness Analyzing Microscope}

Finally, an E-Beam System Specifically Developed For 3D

Surface Roughiness Analysis!

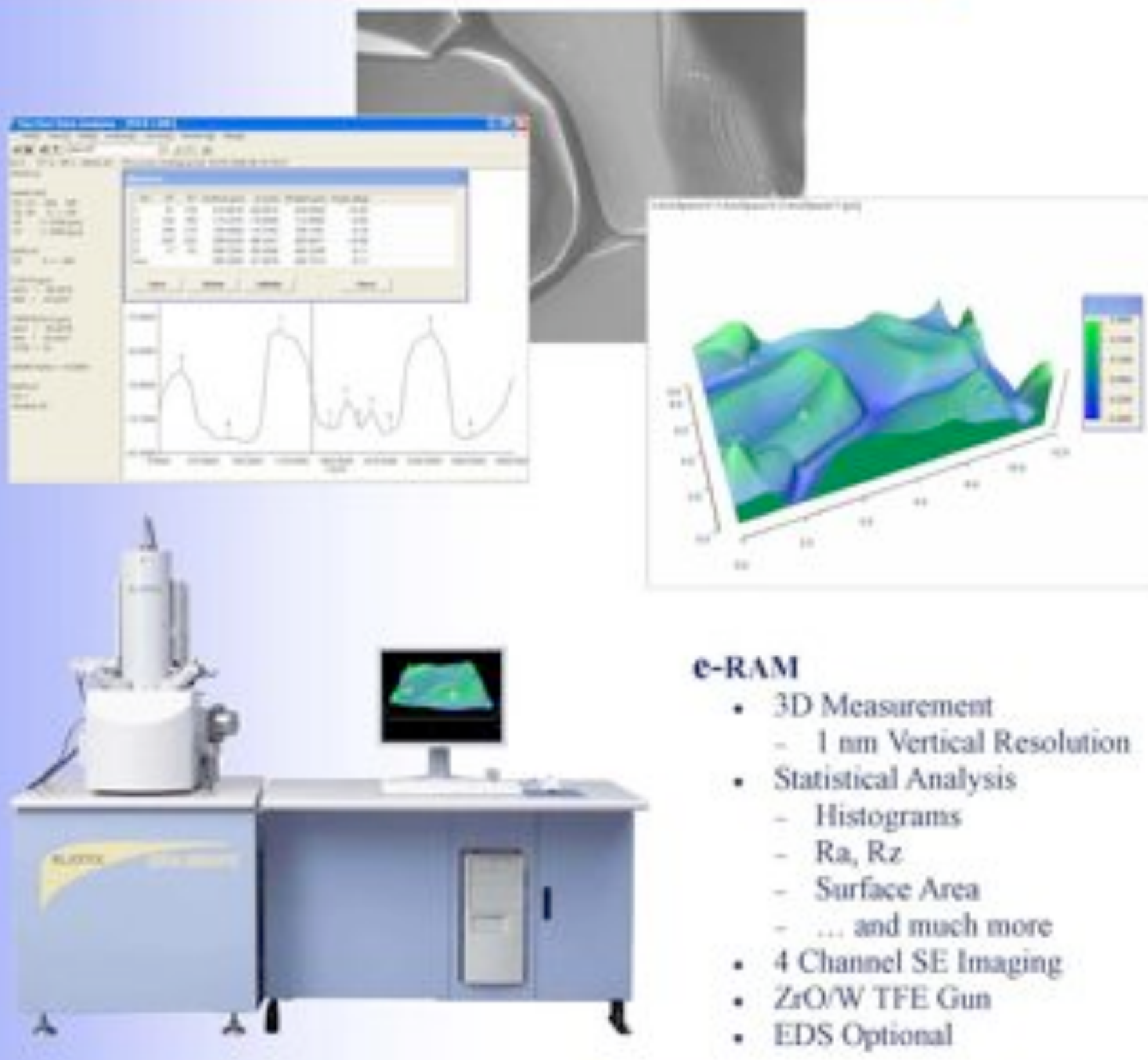

Now that we have your attention... www.sts-elionix.com

\section{STS ELIONIX}




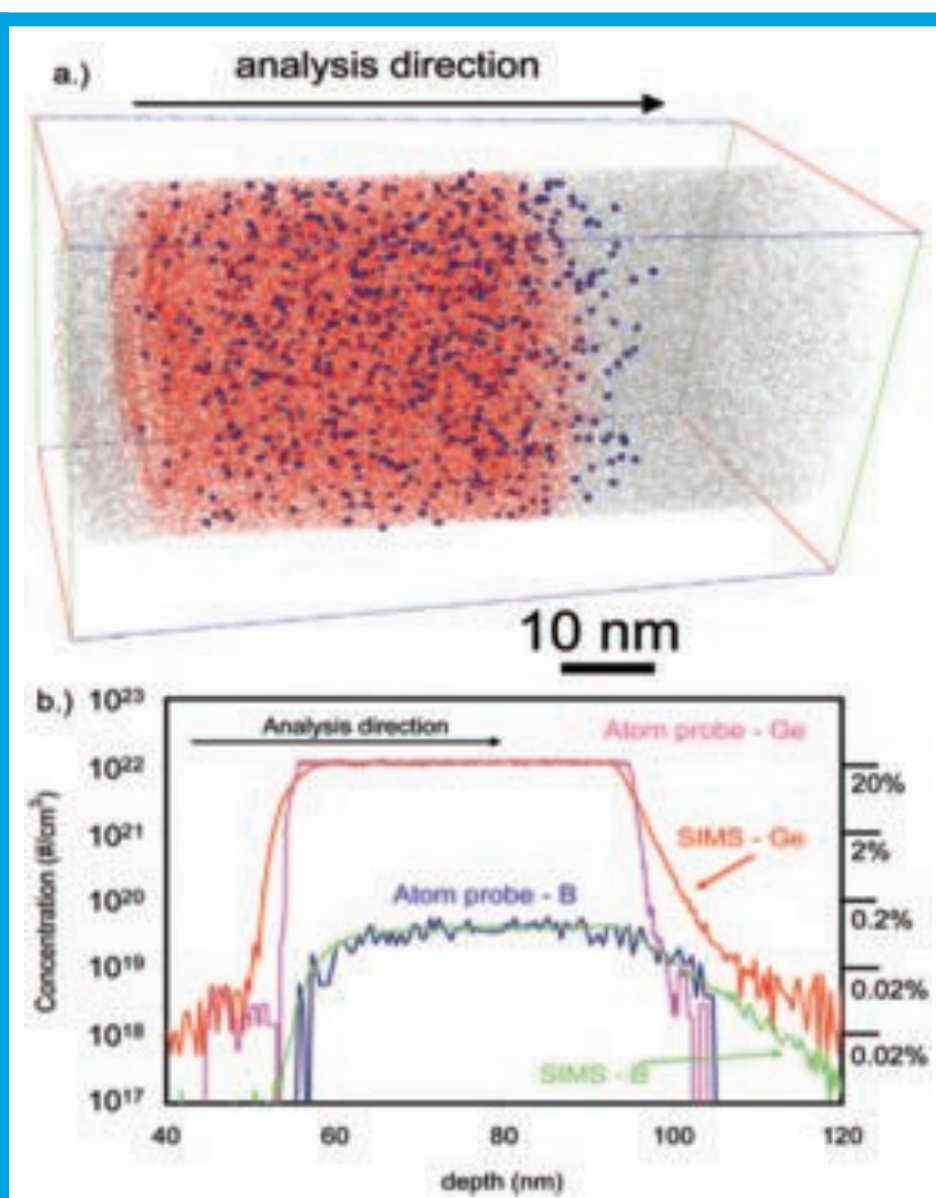

Figure 6a. 3-D atom map of a B-doped SiGe layer. B atoms are rendered as blue spheres, sized larger for emphasis. Si atoms = gray dots. Ge atoms $=$ red dots.

Figure 6b. 1-D composition profile for atom map in Figure $6 a$ along with SIMS correlation.

the stable $\gamma$ phase $(\mathrm{Ag} 2 \mathrm{Al})$ have also nucleated and are growing at the expense of the GP zone as suggested by the Ag-depleted zone. Both are visible in the TEM image (Figure $5 \mathrm{a}$ ) and the atom probe reconstructions (Figure $5 \mathrm{~b}$ and $\mathrm{c}$ ). The concentrations in the GP zones and the $\gamma$ ' platelets are $63 \pm 3$ at. $\% \mathrm{Ag}$ and $66 \pm 2$ at.\% respectively, in agreement with the phase diagram. More generally the temporal evolution of the Ag concentration in the GP zones can be followed and correlated to cluster size. The combination of transmission electron microscopy and atom probe tomography measurements provide key information for connecting to theoretical predictions of the faceting and interfacial sharpness, and provide a basis for explaining the precipitate evolution observed in this system.

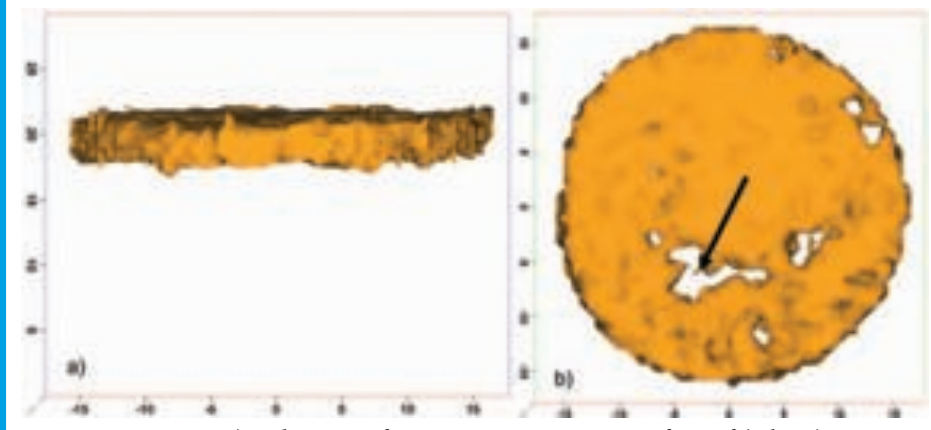

Figure 8. a) Side view of an isoconcentration surface of $(\mathrm{Al}+\mathrm{O})>10 \%$ (similar to plan view TEM image) in a tunneling magnetoresistive barrier device and $b$ ) the same isoconcentration surface shown in plan view.

\section{Si-Ge}

A boron-doped SiGe layer, situated between two undoped Si layers, was analyzed in the LEAP 3000X. This material has an electrical conductivity that is too low for voltage pulsing. The resulting 3-D atom map is shown in Figure 6a where 2\% of the Si atoms (gray dots), 20\% of the Ge atoms (dark black dots) and $100 \%$ of the B dopant atoms (large spheres) are shown. It is evident from the atom map that a small amount of B diffusion, from the SiGe layer into the underlying $\mathrm{Si}$, has occurred. A 1-D composition profile in the analysis direction was produced by creating an analysis cylinder $25 \mathrm{~nm}$ in radius, cutting the cylinder into $1 \mathrm{~nm}$ discs and tabulating the atoms within each slice. On average there were $\sim 25 \mathrm{~B}$ atoms/slice in the SiGe region for a dopant concentration of $3.9 \times 1019 / \mathrm{cm} 3+/-4 \times 1018 / \mathrm{cm} 3$. This compositional analysis is shown in Figure $6 \mathrm{~b}$ along with secondary ion mass spectrometry (SIMS) data for correlation. The SIMS was performed in a $\mathrm{PHI}$ quadrupole system with a $1 \mathrm{keV} \mathrm{Cs}+$ beam at a $60^{\circ}$ angle and a spot size of 25 microns in radius. No oxygen flood was utilized, and an electron flood gun provided charge neutralization. The 1-D composition profiles verify that there was some diffusion of the B into the underlying $\mathrm{Si}$ and also show a slight rarification of $\mathrm{Si}$ towards the top of the SiGe layer. Because the B has diffused into the underlying Si and not into the top Si layer, it is likely that the B diffusion occurred during the $\mathrm{SiGe}$ deposition process.
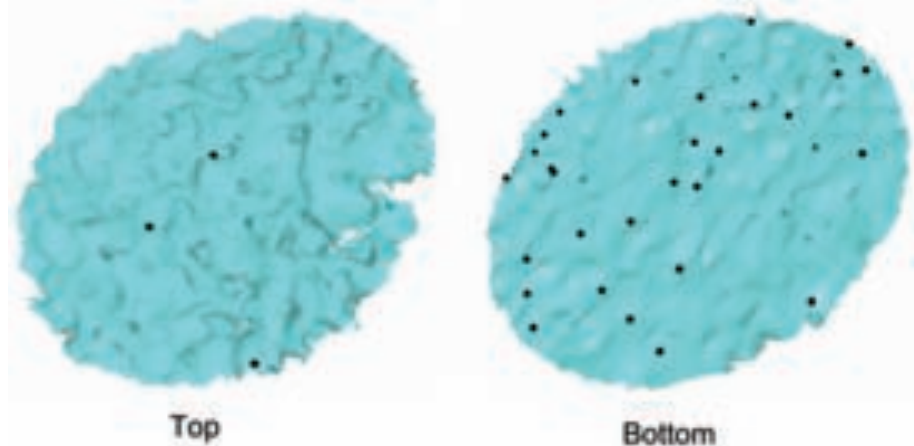

Figure 7. Chemical roughness analysis for Si-SiGe:B-Si system. The top and bottom surfaces had a roughness of $0.47 \mathrm{~nm}$ and $0.26 \mathrm{~nm}$ respectively. The dopant atoms that decorate the surface (i.e. are within $0.5 \mathrm{~nm}$ of the surface) are shown as dark-colored spheres.

In addition to compositional analysis, the knowledge of atom locations allows one to perform a careful analysis of the interface between layers. Specifically, the interface between any two layers was analyzed by constructing an iso-concentration contour across the buried interface for the dataset shown in Figure 6. This isocontour was defined at the point where the local concentration of a given species of atom had risen above or had fallen below a specified value. The average roughness of the interface was then calculated using the ASME B46.1-2002 standard. Because this technique relies upon an analysis of the variation in chemical concentration, it provides a "chemical roughness" - which may be different from the physical roughness measured by atomic force microscopy (AFM).

This chemical roughness calculation was performed for the $\mathrm{Si}$ SiGe:B-Si system, and the associated isocontour maps are shown in Figure 7. The top $\mathrm{Si}-\mathrm{SiGe}$ and bottom $\mathrm{SiGe}-\mathrm{Si}$ interfaces were defined where the Ge concentration crossed the $8 \%$ concentration threshold. In this case, a chemical roughness of $0.47 \mathrm{~nm}$ and $0.26 \mathrm{~nm}$ were calculated at the top $\mathrm{Si}-\mathrm{SiGe}$ and bottom $\mathrm{SiGe}$-Si edges, respectively. In addition, the distribution of $\mathrm{B}$ atoms is shown (dark spheres) across these surfaces. While there is considerable $\mathrm{B}$ decoration along the surface of the bottom edge, there is virtually no dopant decoration of the top edge. This matches well with the 1-D compositional analysis, which shows 


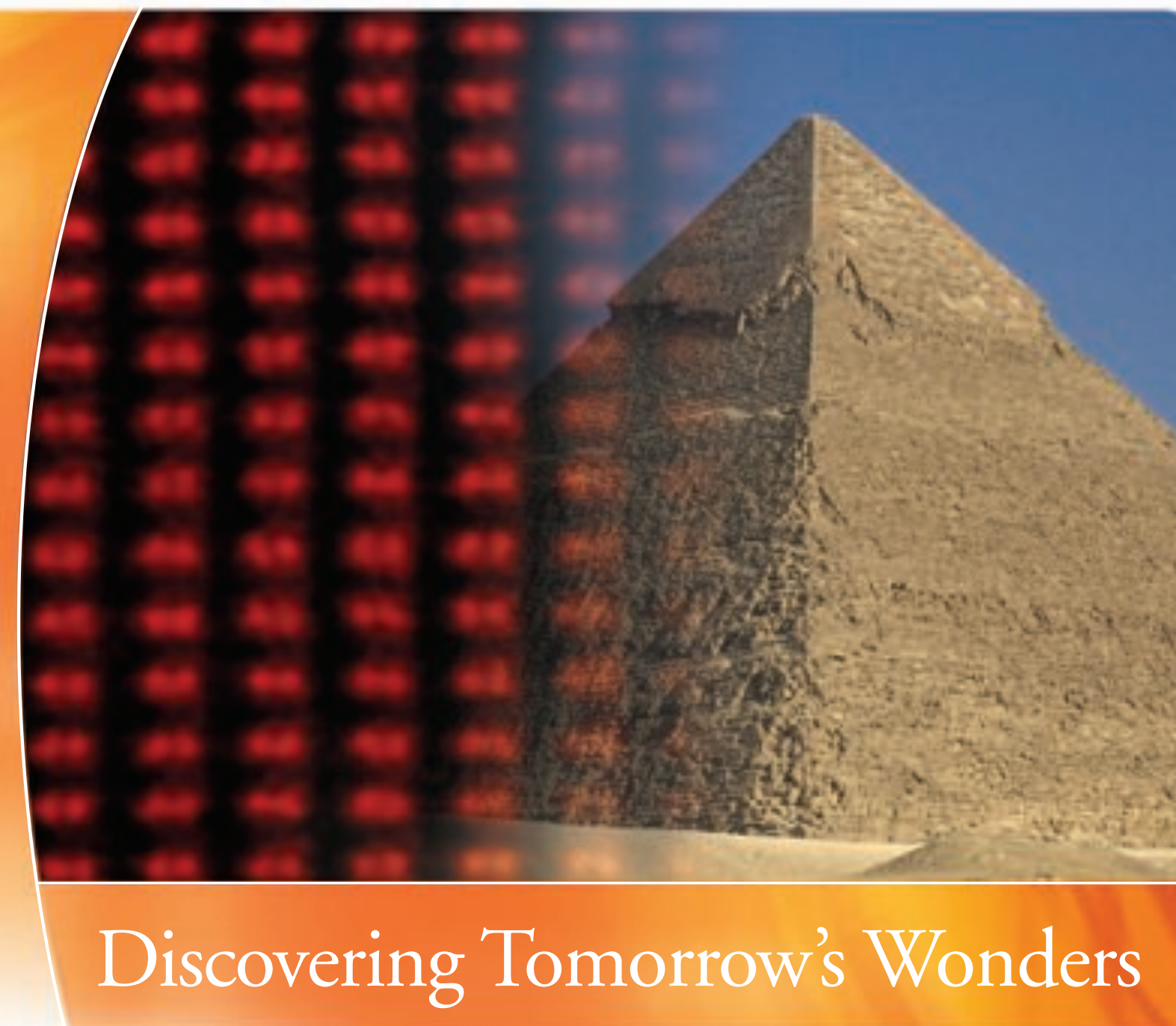

\section{Nanotechnology promises new generations of wonders for our world, impacting virtually every aspect of our lives.}

Since 1966, Fischione Instruments has served the microscopy community with:

\section{TEM/SEM specimen preparation instrumentation \\ Imaging technology \\ TEM specimen holders}

Whether you work in life sciences, physical sciences, or in nanotechnology, our products enable atomic-scale imaging and analysis for genuine insights into the properties and behavior of materials.

Customers worldwide depend on Fischione for innovation, quality, service, and flexibility. Our products support your creativity and give you a clear picture of how materials interact at the most fundamental level — the atomic level.

About the image: Sub-Angstrom image of rows of dumbbell-shaped Si atoms. The specimen was prepared with the Fischione Model 1010 Ion Mill. Information is present down to 0.6 Angstrom. Image courtesy of the Oak Ridge National Laboratory.

The Great Pyramid of Giza, Egypt, one of the wonders of the ancient world. $\underset{I N S T R \cup M}{\text { FISCH }}$ NE 


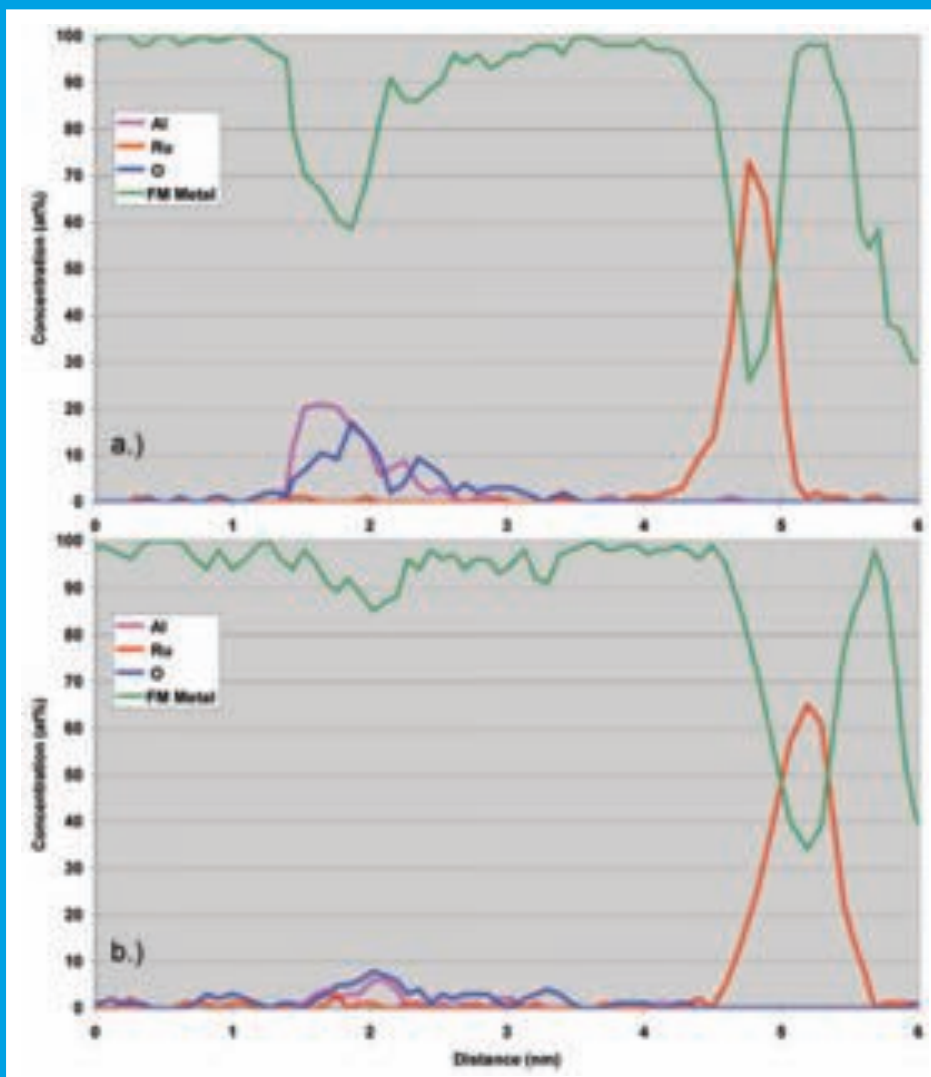

Figure 9. a) Composition profile from a $4 \mathrm{~nm}$ diameter region of $(\mathrm{Al}+\mathrm{O})>\sim 20 \%$ and b) composition profile from a "pinhole" region (shown by arrow in Figure $8 b$ ).

a rarification at the top $\mathrm{Si}-\mathrm{SiGe}$ interface and a migration of dopant atoms into the underlying $\mathrm{Si}$ at the end of the SiGe layer.

\section{Tunneling Magnetoresistive Structures}

Improvements in the properties of nanoscale devices based on giant magnetoresistance (GMR) or tunneling magnetoresistance (TMR) [10] depend on the capabilities of researchers to design, fabricate, and test (i.e. "see") such devices. Disk-drive read heads use thin films whose surfaces are ideally atomically smooth and are exceptionally difficult to analyze. The structure and composition of an aluminum oxide barrier [11] (widely used material for tunnel barriers) and the interfaces between the barrier and the ferromagnetic layers $(\mathrm{Co}, \mathrm{Ni}$, $\mathrm{Fe}$, etc.) play a major role in determining the properties of devices that rely on the TMR effect. The dielectric oxide layer is the crucial component yet is only $1.2 \mathrm{~nm}$ thick. Atom probe tomography analysis of TMR structures provides the positions of the majority of the atoms in such a device to be measured with sub-nm resolution in all three dimensions. Interface attributes such as roughness, composition, and impurities, are critical to performance and can be quantified.

Figure 8 shows a segment of a LEAP reconstruction where an oxide layer from a TMR device structure is delineated by an isoconcentration surface. This type of image is very similar to a cross-sectional transmission electron microscope (TEM) image [11] and suggests a chemically-uniform tunnel barrier. When rotated by 90 degrees, Figure $8 \mathrm{~b}$, this same visualization shows that there are variations in the plane of the barrier that are not visible in cross section. This type of analysis is not possible in TEM because the analytical information is not available in a 3D image. Data such as these are essentially impossible to obtain by any other technique.

In addition to qualitative information on the tunnel barrier, atom probe tomography can provide quantitative chemical analysis of the layers and layer interfaces which comprise the tunnel barrier. Composition profiles through two regions of the barrier shown in Figure 8b are shown in Figure 9. The profile cylinder diameters are $4 \mathrm{~nm}$ and the profile shown in Figure 9a was taken through a region containing high amounts of $\mathrm{Al}$ and $\mathrm{O}$ while the profile in Figure $9 \mathrm{~b}$ was taken through the "pinhole" region [12] shown by the arrow in Figure 8b.

The extremely high spatial resolution of the atom probe technique to quantify the elements in a TMR device is apparent in these figures. The sub-one $\mathrm{nm} \mathrm{Ru}$ layer section of the device, which forms the "synthetic antiferromagnet" portion, is shown on the right side of the composition profiles. There is a clear variation in the quality of the oxide barrier section of the device between the good and bad sections of the barrier, although the good section still reaches only $40 \%(\mathrm{Al}+\mathrm{O})$ concentration. This is, in general agreement with other results in the literature that show significant mixing of the ferromagnetic metals on either side of the tunnel barrier [11,13].

\section{Importance and benefits}

Throughout history, breakthroughs in science have accompanied the introduction of new, more powerful microscopy techniques. For example, the bacteria responsible for the plague were discovered with the optical microscope, and more recently, the SPM was used to discover new atomic-scale surface structure. With its detailed compositional imaging, the LEAP 3000X atom probe will provide unique, valuable information for the nanotechnology revolution that will likely lead to major new discoveries.

Metals have benefited from this sort of information for decades. The advent of commercial laser-pulsed atom probes means that atomic- scale tomography is now available for a very wide spectrum of materials. Furthermore, the possibility of extracting small coupons from materials has greatly simplified and clarified specimen preparation for the technique. The prospects of performing atom probe tomography on so many new materials primes many fields for discoveries through novel analytical information. For example, the 3-D lateral diffusion profile of dopants from the source/drain region into the channel region has recently been accomplished [14]. Simple organic materials have been successfully analyzed [15]. It won't be long before biological components of cells will be studied in the same manner. With such developments, atom probe tomography will not only participate in the mainstream of microscopy, it will set the standard.

\section{References}

[1] A. Cerezo,T.J. Godfrey, S.J. Sibrandij, G.D.W.Smith and P.J. Warren, Rev. Sci. Instrum. 69, 49 (1998)

[2] T. F. Kelly, et al., Micro. Microanal. 10(3) (2004) 373.

[3] K. Thompson, D. J. Larson and R. M. Ulfig, Micro. Microanal. 11(S2) (2005) $882 \mathrm{CD}$.

[4] D. J. Larson, B. D. Wissman, R. J. Viellieux, R. L. Martens, T. T. Gribb, H. F. Erskine, T. F. Kelly and N. Tabat, Micro. Microanal. 7 (2001) 24.

[5] J.E. Gragg and J.B. Cohen, Acta Metall. 19 (1971) 507.

[6] P.A. Dubey, B. Schonfeld, G. Kostorz, Acta Met. et Mater. 39 (1991) 1161.

[7] A. Guinier, Mater. Sci. Forum 217 (1996) 3.

[8] A. Malik, B. Shonfeld, G. Korstorz and J.S. Pederen, Acta Materi. 44 (1996) 4845.

[9] R. Erni, H. Heinrich and G. Kostorz, Phil. Mag. Lett. 83 (2003) 599.

[10] M. Jullière, Phys. Lett. 54A (1975) 225.

[11] A. K. Petford-Long, D. J. Larson, Y. Q. Ma, A Cerezo, B. W. Karr and E. W. Singleton, J. Appl. Phys. 98 (2005) 124904.

[12] D.A. Rabson, B.J. Jönsson-Åkerman, A.H. Romero, R. Escudero, C. Leighton, S. Kim and I.K. Schuller, J. Appl. Phys. 89(5) (2001) 2786.

[13] M. Kuduz et al., Ultramicroscopy 101 (2004) 197.

[14] K. Thompson, J. S. Moore, K. Jones, unpublished research (2006).

[15] T. Prosa, S. Kostrna, T. F. Kelly, unpublished research (2006). 


\section{SEM Like it Hot}

FEl's many commercial SEM firsts have enabled customers to achieve their own groundbreaking results, no matter what the area of investigation.

- First Environmental SEM (ESEM) for imaging and analysis of hot, wet, or uncoated samples

- First SEM to incorporate the Windows graphical user interface

- First integrated SEM/EDS solution

- First large field and gaseous secondary detectors for low vacuum SEM

- First DualBeam ${ }^{\mathrm{Tm}} \mathrm{SEM} / \mathrm{FIB}$ integration

- First high resolution, low vacuum SEM

- First wet STEM option for ESEM

\section{- New Inspect S low vacuum SEM}

Visit us at Microscopy and Microanalysis, booth 304, and see the newest SEM innovations that are enabling today's breakthroughs in research and development. Discover how FEl can be your partner for success.

www.fei.com

sales@feico.com

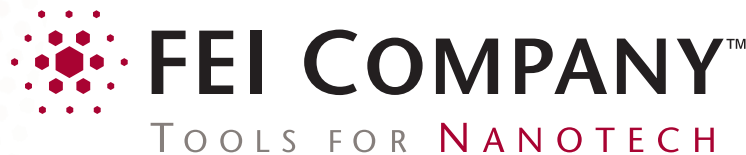
Image of melted glaze at $752^{\circ} \mathrm{C}$, acquired using hot stage on Quanta FEG SEM. C2006 FEl Company. All trademarks are the property of their respective owners.

\section{And the Hottest SEMs Come from FEI}

